\title{
Salidroside inhibits migration, invasion and angiogenesis of MDA-MB 231 TNBC cells by regulating EGFR/Jak2/STAT3 signaling via MMP2
}

\author{
DONG YOUNG KANG ${ }^{1}$, NIPIN SP ${ }^{1}$, DOH HOON KIM ${ }^{1}$, YOUN HEE JOUNG ${ }^{1}$, \\ HYO GUN LEE ${ }^{2}$, YOUNG MIN PARK ${ }^{3}$ and YOUNG MOK YANG ${ }^{1}$
}

\begin{abstract}
${ }^{1}$ Department of Pathology, School of Medicine, Institute of Biomedical Science and Technology, Konkuk University, Chungju, Chungcheongbuk 27478; ${ }^{2}$ Department of Animal Science, College of Natural Resources and Life Sciences, Pusan National University, Miryang, Gyeongsangnam 50463; ${ }^{3}$ Department of Immunology, Lab of Dendritic Cell Differentiation and Regulation, School of Medicine, Konkuk University, Chungju, Chungcheongbuk 27478, Republic of Korea
\end{abstract}

Received February 2, 2018; Accepted May 3, 2018

DOI: $10.3892 /$ ijo.2018.4430

\begin{abstract}
The major hallmarks of tumor progression are angiogenesis, migration and metastasis. Among the components of Rhodiola rosea, salidroside (p-hydroxyphenethyl- $\beta$ - $\mathrm{d}$ glucoside) is one of the most potent, and is present in all Rhodiola species. Recent data have revealed the anticancer effects of salidroside; however, the mechanism underlying its ability to inhibit tumor angiogenesis remains unknown. The present study aimed to analyze how salidroside affects major factors involved in breast cancer, and to elucidate its ability to inhibit angiogenesis and invasion. Signal transducer and activator of transcription 3 (STAT3) is a marker for tumor angiogenesis and migration, which interacts with matrix metalloproteinases (MMPs). Specifically, MMPs act as a downstream target for STAT3. Using western blotting and reverse transcription-quantitative polymerase chain reaction analysis, the present study demonstrated that treatment of MDA-MB 231 triple-negative breast cancer (TNBC) cells with salidroside led to inhibition of invasion and migration markers, and of STAT3 signaling. Furthermore, in vitro angiogenesis analyses in human umbilical vein endothelial cells confirmed the anti-angiogenic activity of salidroside. An electrophoretic mobility shift assay also demonstrated that salidroside may inhibit the DNA-binding activity of STAT3, preventing STAT3 from binding to a novel binding site of the MMP2 gene promoter. In conclusion, the present results demonstrated that
\end{abstract}

Correspondence to: Dr Young Mok Yang, Department of Pathology, School of Medicine, Institute of Biomedical Science and Technology, Konkuk University, 268 Chungwon-daero, Chungju, Chungcheongbuk 27478, Republic of Korea

E-mail: ymyang@kku.ac.kr

Key words: signal transducer and activator of transcription 3, matrix metalloproteinase 2, salidroside, breast cancer, angiogenesis salidroside may downregulate the STAT3 signaling pathway, and inhibit cell viability, migration and invasion through MMPs in breast cancer cells.

\section{Introduction}

Breast cancer is one of the most common types of cancer worldwide, and is a major cause of mortality $(1,2)$. Several types of breast cancer can be differentiated based on receptor expression; $20 \%$ of breast cancer cases express epidermal growth factor receptor (EGFR) and are classified as triplenegative breast cancer (TNBC) (3-5). Notably, EGFR-targeted therapies have been reported to reduce EGFR activity and decrease cancer cell proliferation (6).

All types of breast cancer cells express signal transducer and activator of transcription (STAT) proteins, which serve key roles in cell death, differentiation and proliferation $(7,8)$. STAT3 and STAT5b have important roles in the initiation, progression and metastasis of malignant breast tumors. In particular, STAT3 is strongly associated with cell migration, invasion and metastasis, and its activation is mediated through ligand-associated tyrosine kinases, Janus kinase 2 (Jak2) and EGFR. Phosphorylation of certain STAT3 residues results in the formation of a homodimer or heterodimer that is subsequently translocated to the nucleus. The activated STAT3 molecule acts as a transcription factor in the nucleus, and binds to specific activation elements of the target gene promoter region (9). Previous studies have reported that EGFR, Jak2 and STAT3 signaling has a role in cancer cell proliferation, differentiation, apoptosis and metastasis $(8,10)$.

Rhodiola rosea is widely distributed in high altitude areas, predominantly in Northeast Asia and Eastern Europe, and has long been used as a medicinal material in China. Among the isolated components of $R$. rosea, salidroside (p-hydroxyphenethyl- $\beta$-d-glucoside) is one of the most potent, and is found in all Rhodiola species. Numerous studies have investigated the biological functions of salidroside, highlighting its antioxidant and anticancer effects (11-16). 
Various anticancer investigations have examined the effects of salidroside on the cell cycle and apoptosis; however, to the best of our knowledge, no studies have specifically analyzed the effects of salidroside on breast cancer. The present study investigated how salidroside affected major factors involved in breast cancer, including STAT3, Jak2 and EGFR, in order to elucidate its ability to inhibit metastasis and invasion. The results demonstrated that salidroside treatment downregulated the STAT3 signaling pathway through matrix metalloproteinases (MMPs), thus resulting in the inhibition of cell viability, metastasis, migration and invasion in breast cancer cells.

\section{Materials and methods}

Antibodies and cell culture reagents. Dulbecco's modified Eagle's medium (DMEM) was purchased from Sigma-Aldrich (Merck KGaA, Darmstadt, Germany). Penicillin-streptomycin solution and fetal bovine serum (FBS) were purchased from HyClone (GE Healthcare Life Sciences, Logan, UT, USA). Trypsin-EDTA $(0.05 \%)$ was obtained from Gibco (Thermo Fisher Scientific, Inc., Waltham, MA, USA). Antibodies specific for phosphorylated (p)-STAT3 (Tyr705; sc-7993), STAT1 (sc-464), STAT3 (sc-8019), STAT5b (sc-1656), MMP3 (sc-6839), MMP9 (sc-21733), vascular endothelial growth factor (VEGF; sc-507), VEGF-R2 (sc-6251) and $\beta$-actin (sc-47778), together with secondary antibodies [goat antimouse (sc-2005), anti-rabbit (sc-2004) and donkey anti-goat (sc-2020) immunoglobulin G-horseradish peroxidase (HRP)], were obtained from Santa Cruz Biotechnology, Inc. (Dallas, TX, USA). Anti-TBP (TATA-binding protein; ab818) was purchased from Abcam (Cambridge, UK). An antibody against MMP2 (E90317) was purchased from EnoGene Biotech Co., Ltd. (New York, NY, USA) and an anti-Jak2 (06-1310) antibody was obtained from EMD Millipore (Billerica, MA, USA). Anti-p-Jak2 (\#3776s), p-EGFR (\#2235), EGFR (\#4267), p-STAT1 (\#9167s), p-STAT5 (\#9314) and p-VEGF-R2 (\#2478) antibodies were purchased from Cell Signaling Technology, Inc. (Beverly, MA, USA). Salidroside was purchased from Sigma-Aldrich [Merck KGaA; diluted in dimethyl sulfoxide (DMSO)].

Cell culture and treatment. MDA-MB 231 (no. 30026, Korean Cell Line Bank, Seoul, Korea) human breast cancer cells were cultured in DMEM supplemented with $10 \%$ FBS, $2 \mathrm{mM}$ glutamine, and $100 \mathrm{U} / \mathrm{ml}$ penicillin and streptomycin. MCF-10A immortalized normal human breast epithelial cells [CRL-10317; American Type Culture Collection (ATCC), Manassas, VA, USA] were grown to confluence in phenol redfree DMEM/F12 medium (11320033; Gibco; Thermo Fisher Scientific, Inc.) supplemented with cholera toxin $(1 \mathrm{mg} / \mathrm{ml}$; C8052, Sigma-Aldrich; Merck KGaA), insulin (10 mg/ml; I0516; Sigma-Aldrich; Merck KGaA), EGF (5 mg/ml; E9644; Sigma-Aldrich; Merck KGaA), $1 \%$ penicillin/streptomycin and 5\% FBS. Human umbilical vein endothelial cells (HUVECs; PCS-100-010; ATCC) were maintained in EBM-2 (CC-3156; Lonza, Walkersville, MD USA) endothelial growth basal media. Cells were cultured at $37^{\circ} \mathrm{C}$ in an incubator containing $5 \% \mathrm{CO}_{2}$. For each experiment, cells were resuspended in medium at a density of $2.5 \times 10^{5}$ cells $/ \mathrm{ml}$. Unless otherwise specified, cells were treated with $40 \mu \mathrm{M}$ salidroside for $24 \mathrm{~h}$ at $37^{\circ} \mathrm{C}$.
Cell viability assay. Cell viability was assessed by MTT assay. Briefly, cells were resuspended in DMEM 1 day prior to drug treatment, at a density of $3 \times 10^{3}$ cells/well in 96-well culture plates. Culture medium was replaced with fresh medium containing DMSO as a vehicle control. Cells were incubated with increasing concentrations of salidroside $(5-80 \mu \mathrm{M})$ for $24 \mathrm{~h}$ at $37^{\circ} \mathrm{C}$. Following drug treatment, MTT $(5 \mathrm{mg} / \mathrm{ml})$ was added, and the culture dishes were incubated at $37^{\circ} \mathrm{C}$ for $4 \mathrm{~h}$. The resulting formazan product was dissolved in DMSO and absorbance was measured at $550 \mathrm{~nm}$ on an Ultra Multifunctional Microplate Reader (Tecan US, Inc., Morrisville, NC, USA). All measurements were performed in triplicate, with experiments repeated at least three times.

Western blotting. Whole cell lysates from untreated or salidroside-treated MDA-MB 231 cells and HUVECs were prepared on ice using radioimmunoprecipitation lysis buffer (20-188; EMD Millipore) containing phosphatase and protease inhibitors. Cells were disrupted by aspiration through a 23 -gauge needle and centrifuged at $18,300 \mathrm{x} \mathrm{g}$ for $10 \mathrm{~min}$ at $4^{\circ} \mathrm{C}$ to remove cellular debris. Protein concentrations were measured using the Bradford method (Thermo Fisher Scientific, Inc.). Equal amounts of protein (100 $\mu \mathrm{g} /$ lane) were resolved by $10 \%$ SDS-PAGE and were then transferred onto nitrocellulose membranes. The blots were blocked for $1 \mathrm{~h}$ with $5 \%$ skimmed milk in TBS-T buffer [20 mM Tris-HCl (pH 7.6), $137 \mathrm{mM} \mathrm{NaCl}, 0.1 \mathrm{X}$ Tween-20]. Membranes were then probed overnight at $4^{\circ} \mathrm{C}$ with the relevant primary antibodies (MMP2, MMP3 and MMP9, 1:500 dilutions; STAT1, STAT3, STAT5b, p-STAT1, p-STAT3, p-STAT5, VEGF, VEGF-R2, p-Jak2, Jak2, p-EGFR, EGFR, p-VEGF-R2, TBP and $\beta$-actin, 1:1,000 dilutions) diluted in 5\% bovine serum albumin (BSA; EMD Millipore) or skim milk (Difco ${ }^{\mathrm{TM}}$ Skim Milk; BD Biosciences, Franklin Lakes, NJ, USA), followed by washing with TBS-T and incubation for $1 \mathrm{~h}$ at room temperature with HRP-conjugated secondary antibodies (1:2,000 dilution in $5 \%$ BSA or skim milk). Detection was performed using the Enhanced Chemiluminescence Plus detection kit (Amersham; GE Healthcare, Chicago, IL, USA) and an LAS-4000 imaging device (Fujifilm, Tokyo, Japan). Blots were stripped using Restore Western Blot Stripping Buffer (Thermo Fisher Scientific, Inc.).

Reverse transcription-polymerase chain reaction (RT-PCR). Total RNA was extracted using the RNeasy Mini kit (Qiagen $\mathrm{GmbH}$, Hilden, Germany) according to the manufacturer's protocol. RNA was quantified spectrophotometrically at $260 \mathrm{~nm}$. Subsequently, RT-PCR analyses were performed to detect MMP2, MMP3, MMP9 and 18s RNA expression. cDNA was synthesized from total RNA at $42^{\circ} \mathrm{C}$ for $1 \mathrm{~h}$ and $95^{\circ} \mathrm{C}$ for 5 min using first-strand cDNA synthesis kits (K-2041; Bioneer Corporation, Daejeon, Korea) and oligo d(T) primers. The RT-PCR Premix kit (K-2016; Bioneer Corporation) was used for MMP2, MMP3 MMP9 and 18s amplification with primers synthesized by Bioneer Corporation. PCR amplification to generate a 472-bp MMP2 fragment was conducted with the following primers: MMP2, sense 5'-GGCCCTGTCAC TCCTGAGAT-3' and antisense 5'-GGCATCCAGGTTATCGG GGA-3'. The PCR conditions were as follows: $94^{\circ} \mathrm{C}$ for $5 \mathrm{~min}$, followed by 32 cycles at $94^{\circ} \mathrm{C}$ for $30 \mathrm{sec}, 58^{\circ} \mathrm{C}$ for $30 \mathrm{sec}$ and 
$72^{\circ} \mathrm{C}$ for $45 \mathrm{sec}$, followed by $72^{\circ} \mathrm{C}$ for $7 \mathrm{~min}$. PCR amplification to generate a 432-bp MMP3 fragment was conducted with the following primers and PCR conditions: MMP3, sense 5'-CCT GCTTTGTCCTTTGATGC-3' and antisense 5'-TGAGTCAA TCCCTGGAAAGT-3'; $95^{\circ} \mathrm{C}$ for $5 \mathrm{~min}$, followed by 32 cycles at $95^{\circ} \mathrm{C}$ for $45 \mathrm{sec}, 60^{\circ} \mathrm{C}$ for $45 \mathrm{sec}$ and $72^{\circ} \mathrm{C}$ for $60 \mathrm{sec}$, followed by $72^{\circ} \mathrm{C}$ for $10 \mathrm{~min}$. PCR amplification to generate a 455 -bp MMP9 fragment was conducted with the following primers and PCR conditions: MMP9, sense 5'-CCTGCCAGTTTCC ATTCATC-3' and antisense 5'-GCCATTCACGTCGTCCT TAT-3'; $94^{\circ} \mathrm{C}$ for $5 \mathrm{~min}$, followed by 30 cycles at $94^{\circ} \mathrm{C}$ for $40 \mathrm{sec}, 60^{\circ} \mathrm{C}$ for $40 \mathrm{sec}$ and $68^{\circ} \mathrm{C}$ for $50 \mathrm{sec}$, followed by $72^{\circ} \mathrm{C}$ for $7 \mathrm{~min}$. Finally, a 489-bp amplified 18s mRNA fragment was generated using the following primers and PCR conditions: 18s, sense 5'-CGGCTACCACATCCAAGGAA-3' and antisense 5'-CCGGCGTCCCTCTTAATC-3'; $95^{\circ} \mathrm{C}$ for $5 \mathrm{~min}$, followed by 30 cycles at $95^{\circ} \mathrm{C}$ for $60 \mathrm{sec}, 58^{\circ} \mathrm{C}$ for $60 \mathrm{sec}$ and $72^{\circ} \mathrm{C}$ for $60 \mathrm{sec}$, followed by $72^{\circ} \mathrm{C}$ for $10 \mathrm{~min}$. PCR products were resolved by electrophoresis on a $2 \%$ agarose gel and were visualized by ethidium bromide (E7637; Sigma-Aldrich; Merck KGaA) staining.

Electrophoretic mobility shift assay (EMSA). STAT3 DNA-binding activity was detected using LightShift Chemiluminescent EMSA kit (20148; Thermo Fisher Scientific, Inc.). Oligonucleotide probes (STAT3) and reporter lysis buffer were purchased from Promega Corporation (Madison, WI, USA). MDA-MB 231 cells were grown to $\sim 90 \%$ confluence with nuclear protein extracts prepared using the Nuclear Extract kit (Affymetrix; Thermo Fisher Scientific, Inc.). EMSA was performed using the EMSA kit (20148; Thermo Fisher Scientific, Inc.), according to the manufacturer's protocol. Briefly, nuclear protein was subjected to hybridization to a double-stranded, biotin-labeled oligonucleotide probe, containing the consensus-binding site for STAT3 (sense strand, 5'-GATCCTTCTGGGAATTCCTAGATC-3'). Proteins were resolved on a non-denaturing 6\% PAGE gel (Bio-Rad Laboratories, Inc., Hercules, CA, USA). Proteins were transferred from the gel to a nylon membrane and detected using streptavidin-HRP and a chemiluminescent substrate (Affymetrix; Thermo Fisher Scientific, Inc.).

Small interfering RNA (siRNA) analyses. MDA-MB 231 cells $(1 \times 105)$ were cultured in 6 -well plates, and grown to $60 \%$ confluence. Cells were then transfected with the ON-TARGETplus SMARTpool siRNA targeting STAT3 (siSTAT3), or ON-TARGETplus non-target siRNA (GE Healthcare Dharmacon, Inc., Lafayette, CO, USA), as a control. Transfection was conducted using the DharmaFECT transfection reagent (GE Healthcare Dharmacon, Inc.), according to the manufacturer's protocol. Following siRNA transfection $(1 \mu \mathrm{g} / \mu \mathrm{l})$ for $24 \mathrm{~h}$ at $37^{\circ} \mathrm{C}$, salidroside treatment was applied for an additional $24 \mathrm{~h}$. The expression levels of STAT3, p-STAT3, MMP2 and $\beta$-actin were detected by western blotting.

Wound healing assay. MDA-MB 231 cells were cultured in 6 -well plates at a concentration of $1 \times 10^{5}$ cells/well in DMEM containing $10 \%$ FBS and were then incubated for $24 \mathrm{~h}$ in a humidified chamber. After forming a confluent monolayer, the cell layer was scratched with a pipette tip and washed with PBS to remove cell debris. Cells were untreated (controls) or exposed to 10, 20 and $40 \mu \mathrm{M}$ salidroside. Images of the scratch sites (wounds) were captured at 0 and $24 \mathrm{~h}$ using a fluorescence microscope (IX71; Olympus Corporation, Center Valley, PA, USA), and the average area of the wound was calculated using DP Controller software (Version 3.2; Olympus Corporation).

Matrigel invasion assay. A Transwell invasion assay was performed using Matrigel pre-coated, ready-to-use invasion chambers (BD BioCoat; BD Biosciences). MDA-MB 231 cells in DMEM, suspended at a concentration of $5 \times 10^{4}$ were added to the inserts. Drug-containing $(10,20$ and $40 \mu \mathrm{M})$ media were added to the receiver plate, and the inserts containing cells were placed onto it. After 24-h incubation in a humidified chamber at $37^{\circ} \mathrm{C}$, the cells that had invaded the apical surface of the inserts were identified using crystal violet. The plates were then incubated in ambient conditions for $24 \mathrm{~h}$, after which they were washed and fixed with $3.7 \%$ formaldehyde for $5 \mathrm{~min}$ at room temperature. The cells on the upper surface were removed using a cotton swab and the invaded cells were quantified under a fluorescence microscope. Cells were counted in four fields of view.

In vitro angiogenesis assay. ECMatrix (In Vitro Angiogenesis Assay kit; ECM625; EMD Millipore) was thawed at $4^{\circ} \mathrm{C}$ overnight, after which the wells of pre-chilled 96 -well plates were coated with $50 \mu \mathrm{l}$ diluted ECMatrix and incubated at $37^{\circ} \mathrm{C}$ for $1 \mathrm{~h}$. A total of $150 \mu \mathrm{l}$ HUVECs $\left(1 \times 10^{4}\right.$; American Type Culture Collection; CRL-1730) with or without salidroside was added to the solidified matrix and incubated at $37^{\circ} \mathrm{C}$ for $12 \mathrm{~h}$. Endothelial cell formation was observed under a fluorescence microscope. Focus was placed on distinct areas and the tubes formed were counted.

Transfection and STAT3 overexpression analysis. MDA-MB 231 cells $\left(1 \times 10^{5}\right)$ were cultured in 6-well plates and grown to $60 \%$ confluence. The cells were then transfected with STAT3-pMX vector (provided by Dr M. Shong, Chungnam National University, Daejeon, Korea) or empty pMX vector (control, $0.5 \mu \mathrm{g} / \mu \mathrm{l}$ ) using the DharmaFECT transfection reagent (GE Healthcare Dharmacon, Inc.) for $24 \mathrm{~h}$ at $37^{\circ} \mathrm{C}$. Transfected cells were washed with ice-cold PBS, and treated for an additional $24 \mathrm{~h}$ with media that contained $40 \mu \mathrm{M}$ salidroside. Proteins were isolated and analyzed by western blotting to determine p-STAT3, STAT3, MMP2 and $\beta$-actin expression levels.

Molecular docking. The binding of salidroside to EGFR was determined by molecular docking using the AutoDock Vina program in PyRx software version 0.8 (17). The 3D structure of salidroside was obtained from PubChem (ID: 159278; https:// pubchem.ncbi.nlm.nih.gov/) and that of EGFR was obtained from Protein Data Bank (PDB ID: 2GS2; https://www.rcsb. org/). The obtained binding was analyzed by PyMol software version 0.99 (https://pymol.en.uptodown.com/).

Statistical analyses. All experiments were performed at least three times with results expressed as means \pm standard error of the mean. Statistical analyses were conducted using one-way 
$\Delta$
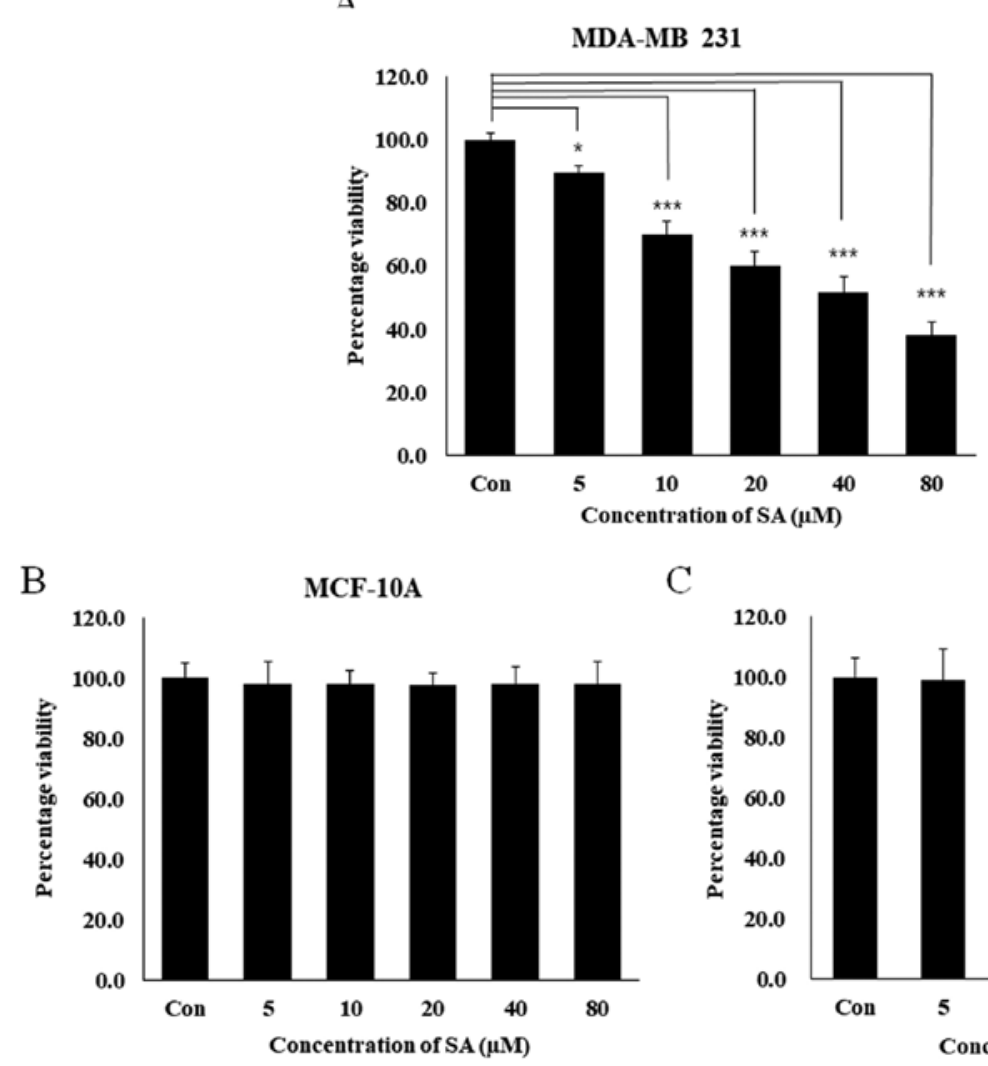

B

$\mathrm{C}$

HUVEC

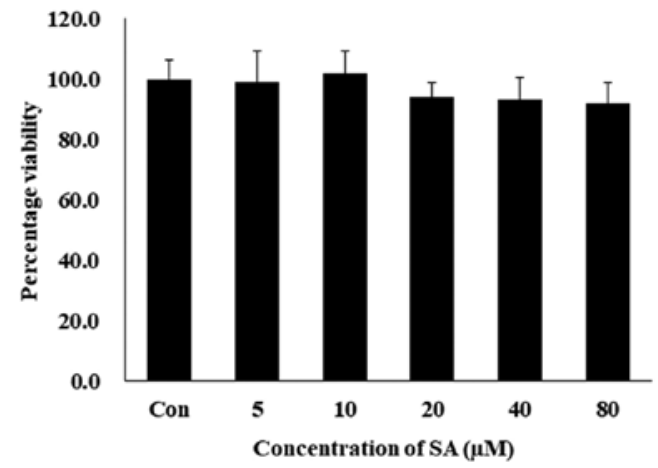

Figure 1. SA dose-dependently inhibits the viability of MDA-MB 231 breast cancer cells. (A) MDA-MB 231, (B) MCF-10A and (C) HUVECs were exposed to SA for $24 \mathrm{~h}$, and cell viability was evaluated by MTT assay. SA at a concentration of $40 \mu \mathrm{M}$ resulted in $50 \%$ inhibition of MDA-MB 231 cell viability. HUVECs, human umbilical vein endothelial cells; SA, salidroside. Statistical analyses were performed using analysis of variance. ${ }^{*} \mathrm{P}<0.05$, ${ }^{, * * *} \mathrm{P}<0.001 \mathrm{com}-$ pared with the control.

analysis of variance (ANOVA) or Student's t-test. One-way ANOVA was performed with Duncan's multiple range test as a post hoc test. Analyses were performed using the SAS 9.3 program (SAS Institute, Inc., Cary, NC, USA). P<0.05 was considered to indicate a statistically significant difference.

\section{Results}

Salidroside inhibits MDA-MB 231 cell viability. MTT assay was performed to examine how salidroside affected the viability of the human breast cancer cell lines MDA-MB 231 and MCF-10A. For 24 h, MDA-MB 231, MCF-10A and HUVECs were exposed to increasing concentrations of salidroside (5, $10,20,40$ and $80 \mu \mathrm{M})$. The numbers of salidroside-treated cells compared with control cells were then compared during the logarithmic growth phase. MDA-MB 231 cell growth was inhibited by $49 \%$ following treatment with $40 \mu \mathrm{M}$ salidroside, and by $62 \%$ following treatment with $80 \mu \mathrm{M}$ salidroside (Fig. 1A, P<0.001). Conversely, salidroside did not inhibit the viability of normal cell lines, MCF-10A and HUVECs (Fig. 1B and C). Salidroside treatment markedly decreased the viability of MDA-MB 231 cells in a dose-dependent manner, with $40 \mu \mathrm{M}$ salidroside determined to be the half maximal inhibitory concentration; this concentration was used in subsequent experiments.

Salidroside inhibits angiogenesis through VEGF and STAT3 in HUVEC cells. To confirm that salidroside exerted inhibitory effects on angiogenesis, an in vitro angiogenesis assay was conducted using 40 and $80 \mu \mathrm{M}$ salidroside (Fig. 2A). Salidroside significantly inhibited tube formation in the extracellular matrix (Fig. 2B). VEGF serves an important role in angiogenesis. Western blot analysis confirmed that treatment with $40 \mu \mathrm{M}$ salidroside inhibited the expression levels of VEGF and p-STAT3 in HUVECs without altering the levels of total STAT3 (Fig. 2C and D). These results suggested that salidroside may inhibit VEGF-dependent angiogenesis through STAT3.

Salidroside suppresses EGFR phosphorylationandJak2/STAT3 signaling in MDA-MB 231 cells. The EGF-initiated Jak and STAT signaling cascade has been implicated in cell survival responses; Jak phosphorylates STAT proteins localized at the plasma membrane $(18,19)$. The present study evaluated the binding ability of salidroside with EGFR using molecular docking with the AutoDock Vina platform. Salidroside docked with the ATP-binding site of EGFR, thus confirming direct binding of salidroside with EGFR (Fig. 3A). Western blot analysis revealed that salidroside treatment led to downregulation of p-EGFR. These results suggested that salidroside treatment may inhibit EGFR binding, and thus downregulate the expression of its downstream molecules p-Jak2 and p-STAT3. Compared with in the control group, treatment with $40 \mu \mathrm{M}$ salidroside resulted in a reduction in the expression of these proteins (Fig. 3B). These data suggested that salidroside may inhibit EGFR phosphorylation, which resulted in reduced 
A

$$
\text { Con }
$$
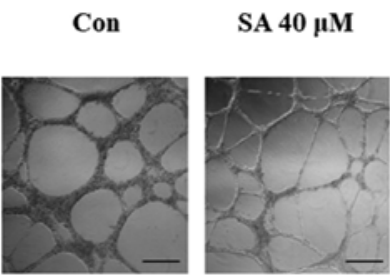

B

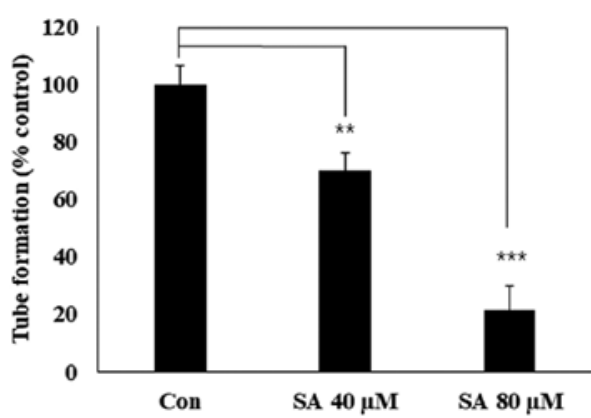

C HUVEC

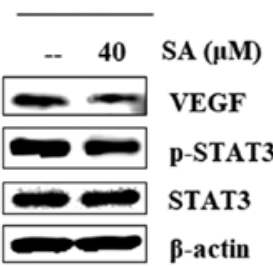

D

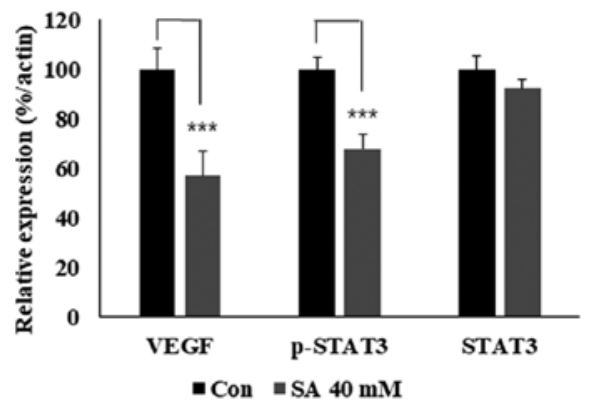

Figure 2. SA inhibits angiogenesis in HUVECs. (A) In vitro angiogenesis assay revealed that SA inhibited angiogenesis in HUVEC cells (scale bars: $100 \mu \mathrm{m}$ ). (B) Graphical representation of the in vitro angiogenesis assay showing relative inhibition of tube formation. Statistical analyses were performed using Student's t-test. ${ }^{* *} \mathrm{P}<0.01,{ }^{* * *} \mathrm{P}<0.001$. (C) Western blot analysis revealed that VEGF and p-STAT3 were inhibited in HUVECs treated with $40 \mu \mathrm{M}$ SA for $24 \mathrm{~h}$. (D) Relative protein expression levels of VEGF, p-STAT3 and STAT3 were determined using densitometry; levels were normalized to $\beta$-actin. Data are representative of three independent experiments. Statistical analyses were performed using Student's t-test. ${ }^{* * *} \mathrm{P}<0.001$. HUVECs, human umbilical vein endothelial cells; p, phosphorylated; SA, salidroside; STAT3, signal transducer and activator of transcription 3; VEGF, vascular endothelial growth factor.

A

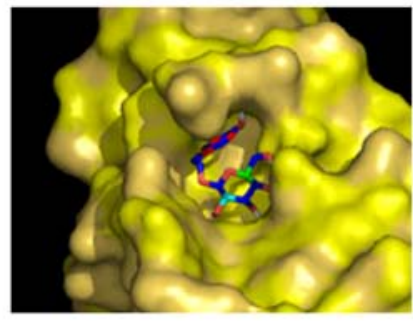

B

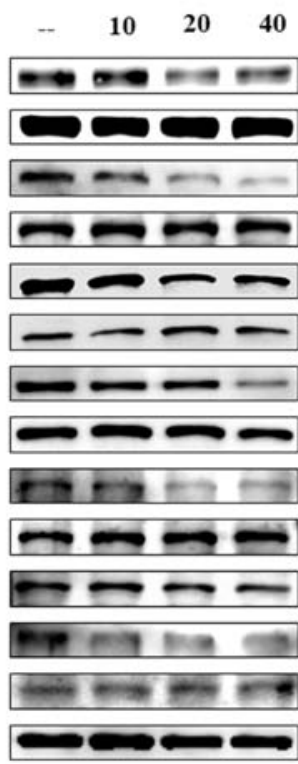

EGFR

p-Jak2

Jak2

STAT1

STAT3

STAT5b

VEGF

VEGF-R2

$\beta$-actin
C

\section{Nuclear extracts}

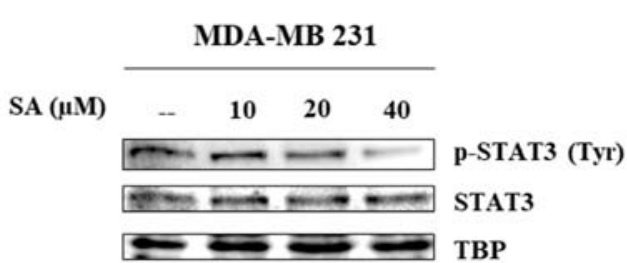

D

$\mathrm{SA} 40(\mu \mathrm{M})$

p-STAT1 (Tyr)

p-STAT3 (Tyr)

p-STAT5 (Tyr)

p-VEGF-R2

Figure 3. SA inhibits EGFR/Jak2/STAT signaling activity and nuclear translocation, as well as the DNA-binding activity of STAT3. (A) Binding of SA (PubChem ID: 159278) to the ATP-binding domain of EGFR (Protein Data Bank ID: 2GS2), as determined by molecular docking using AutoDock Vina. (B) Western blot analysis revealed inhibition of EGFR activity and STAT3 signaling in MDA-MB 231 cells following treatment with increasing concentrations of SA for $24 \mathrm{~h}$. (C) MDA-MB 231 cells were exposed to 10-40 $\mu \mathrm{M}$ salidroside for $24 \mathrm{~h}$, and nuclear proteins were analyzed by western blotting, with TBP as a loading control. SA inhibited nuclear translocation of STAT3. (D) Gel shift analysis of nuclear extracts revealed that salidroside treatment inhibited the DNA-binding activity of STAT3 to the $\gamma$-interferon activation site element. EGFR, epidermal growth factor receptor; Jak2, Janus kinase 2; p, phosphorylated; SA, salidroside; STAT, signal transducer and activator of transcription; TBP, TATA-binding protein; VEGF, vascular endothelial growth factor; VEGF-R2, VEGF receptor 2. 
A

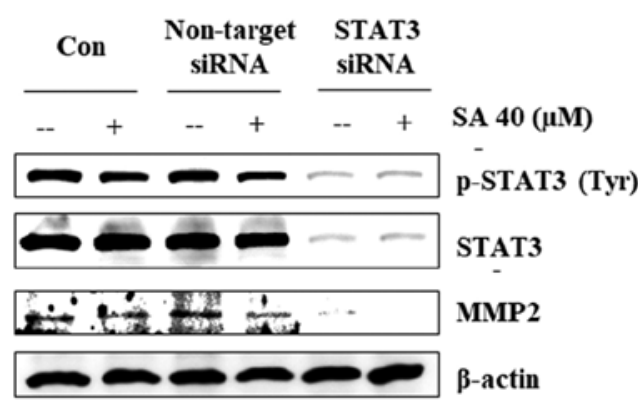

$\mathrm{C}$

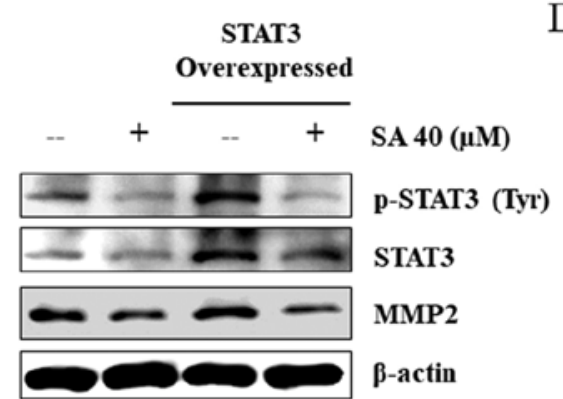

$\mathrm{D}$
$\mathrm{B}$
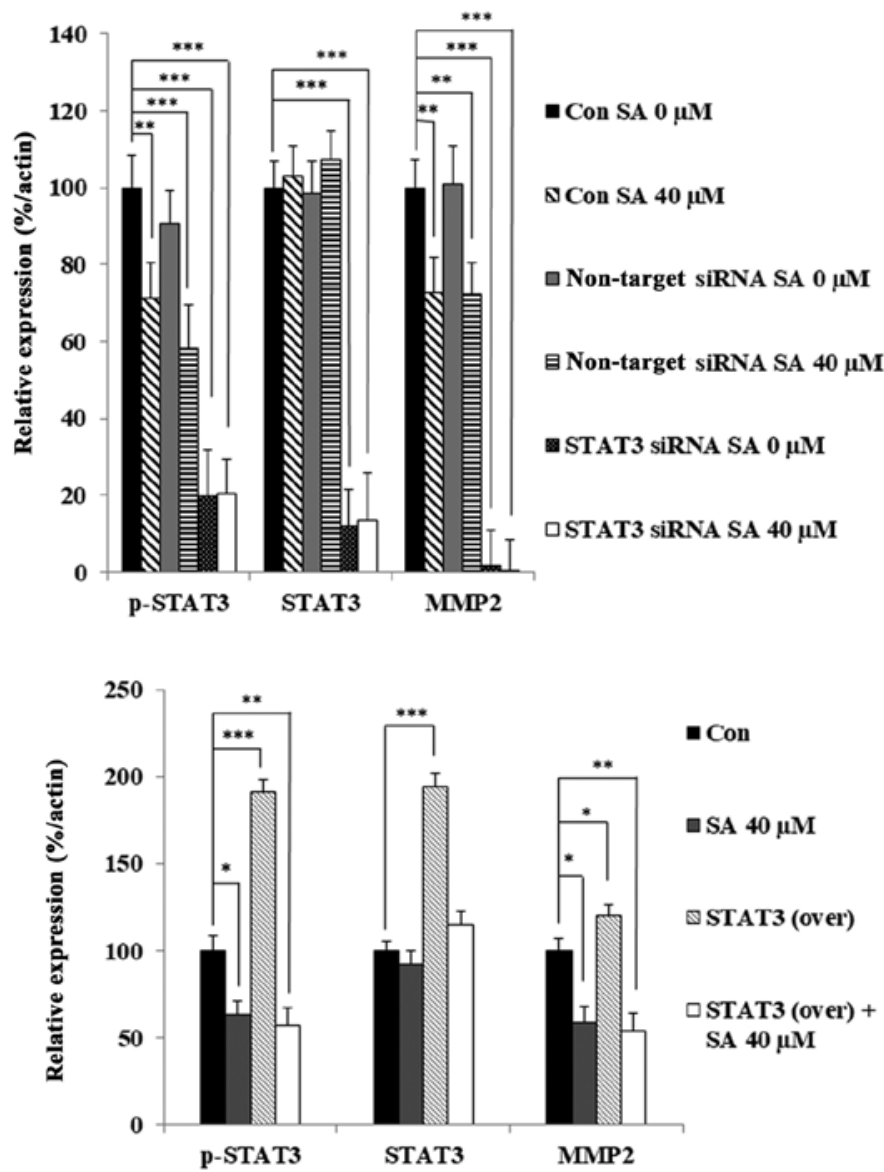

Figure 4. STAT3 regulates MMP2 expression in MDA-MB 231 cells upon SA treatment. (A) Targeted STAT3 silencing decreased MMP2 expression in MDA-MB 231 cells. (B) Relative protein expression levels of p-STAT3, STAT3 and MMP2 were determined using densitometry, and were normalized to $\beta$-actin. Data are representative of three independent experiments. Statistical analyses were performed using one-way analysis of variance. ${ }^{* *} \mathrm{P}<0.01,{ }^{* * *} \mathrm{P}<0.001$ compared with the control. (C) STAT3-overexpressing MDA-MB 231 cells exhibited decreased p-STAT3 and MMP2 protein expression following treatment with $40 \mu \mathrm{M}$ SA. (D) Relative levels of p-STAT3, STAT3, and MMP2 proteins were determined using densitometry, and normalized $\beta$-actin. Statistical analyses were performed using one-way analysis of variance. ${ }^{*} \mathrm{P}<0.05,{ }^{* * *} \mathrm{P}<0.01,{ }^{* * *} \mathrm{P}<0.001$. Data are representative of three independent experiments. MMP2, matrix metalloproteinase 2; p, phosphorylated; SA, salidroside; siRNA, small interfering RNA; STAT3, signal transducer and activator of transcription 3.

phosphorylation of Jak2 and STAT3. Furthermore, inhibition of the angiogenesis markers VEGF and p-VEGF-R2 confirmed that salidroside inhibited angiogenesis.

Salidroside inhibits nuclear translocation and DNA-binding activity of STAT3. A recent study reported that STAT3 activation regulates MMP2 expression (20). MMP2 transcription requires translocation of p-STAT3 to the nucleus, where it binds to the MMP2 gene promoter. To analyze nuclear translocation, nuclear extracts were isolated from untreated and salidroside-treated MDA-MB 231 cells. Western blot analysis of these nuclear extracts revealed that the expression levels of p-STAT3 were reduced in salidroside-treated cells (Fig. 3C). In our previous study, the MMP2 gene promoter was analyzed, in order to detect a $\gamma$-interferon activation site (GAS) element (20), which is the DNA-binding sequence for the STAT family of transcription factors. Upon locating this element, an EMSA was conducted to analyze the DNA-binding activity of STAT3 to the GAS element. The results indicated that the DNA/STAT3 complex was downregulated in salidroside-treated cells compared with in the untreated control cells (Fig. 3D). These findings revealed that salidroside may inhibit expression of the STAT3/MMP2 complex, thus indicating that salidroside acts through STAT3 signaling.

Salidroside suppresses MMP2 expression via STAT3 regulation. Upon confirming the downregulation of MMP2 expression, and the downregulation of STAT3 expression, phosphorylation and DNA-binding activity, the present study investigated the association between STAT3 and MMP2. To test the hypothesis that STAT3 may be a positive regulator of MMP2, STAT3 was knocked down using specific siSTAT3, and MMP2 expression was analyzed by western blotting. The results verified the role of STAT3 in MMP2 transcription. Among salidroside-treated cells, MMP2 expression was reduced in cells targeted with siSTAT3 compared with in cells transfected with non-targeting siRNA (Fig. 4A). Analyzing the protein expression levels relative to $\beta$-actin clearly elucidated the impact of salidroside on STAT3-related MMP2 expression (Fig. 4B).

STAT3 protein was subsequently overexpressed in MDA-MB 231 cells, and STAT3 and MMP2 expression were analyzed using western blotting (Fig. 4C). Salidroside treatment led to $\sim 40 \%$ inhibition of p-STAT3 and MMP2 expression in MDA-MB 231 cells compared with in the 
A
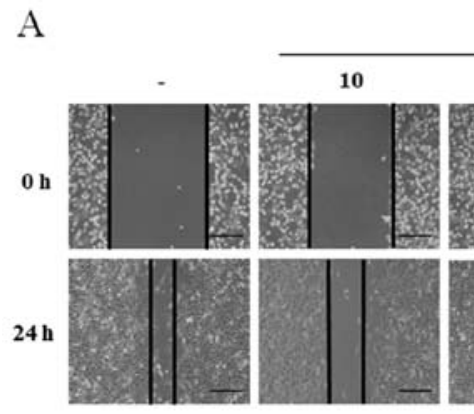

C

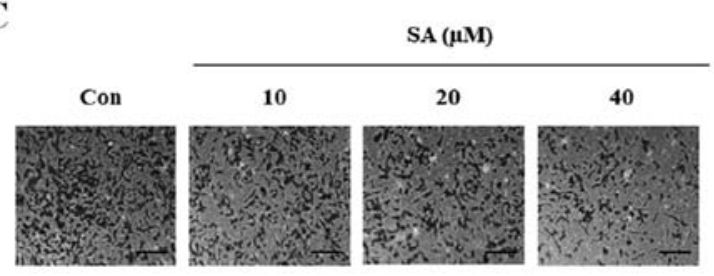

B

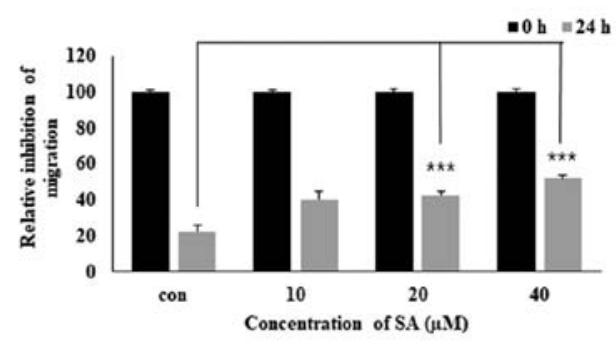

$\mathrm{D}$

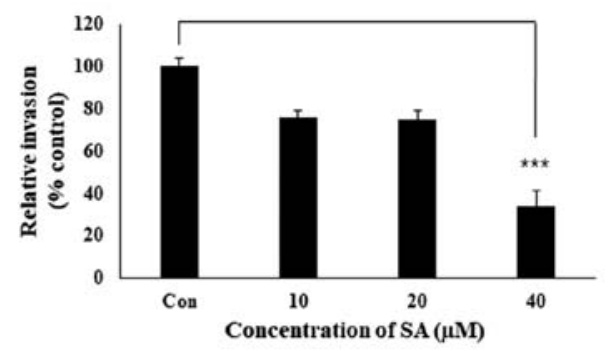

$\mathrm{E}$

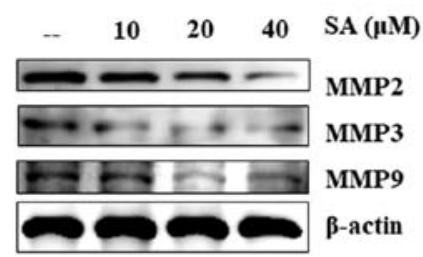

$\mathrm{F}$

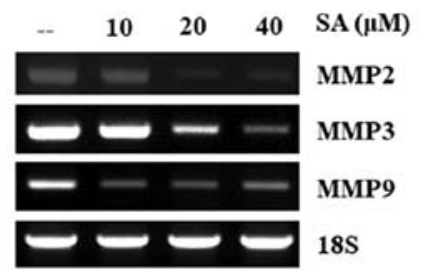

Figure 5. SA inhibited the migration and invasion of MDA-MB 231 cells. (A) Wound-healing assay in MDA-MB 231 cells revealed that migration was inhibited following 24-h treatment with SA (scale bars: $100 \mu \mathrm{m}$ ). (B) SA induced relative inhibition of migration in MDA-MB 231 cells. Statistical analyses were performed using one-way analysis of variance. ${ }^{* * *} \mathrm{P}<0.001$. (C) Matrigel invasion assay demonstrated that after $24 \mathrm{~h}, \mathrm{SA}$ inhibited the invasive ability of MDA-MB 231 cells (scale bars: $100 \mu \mathrm{m}$ ). (D) Relative invasion in MDA-MB 231 cells. Data are representative of three independent experiments. Statistical analyses were performed using Student's t-test. ${ }^{* * *} \mathrm{P}<0.001$. (E) Western blot analysis revealed that after 24 -h treatment with SA, the expression levels of MMP2, MMP3, and MMP9 were reduced in MDA-MB 231 cells. (F) Reverse transcription-polymerase chain reaction analysis of MMP2, MMP3 and MMP9 in MDA-MB 231 cells after 24-h salidroside treatment. MMP, matrix metalloproteinase; SA, salidroside.

untreated control cells. In STAT3-overexpressing cells, salidroside treatment led to 55\% inhibition of MMP2 compared with untreated STAT3-overexpressing MDA-MB 231 cells (Fig. 4D). Analysis of cell lysates revealed similar expression patterns of p-STAT3 and MMP2 following salidroside treatment, indicating that salidroside suppressed STAT3 and MMP2 expression. Based on these results, it may be concluded that STAT3 serves an essential role in MMP2 activation.

Salidroside suppresses cell migration and cellular invasion. Migration and invasion are considered key regulatory processes in tumor progression, angiogenesis and metastasis. Therefore, the present study analyzed whether salidroside inhibited cell migration and invasion via its effects on MMPs and STAT3. A wound-healing assay was performed in MDA-MB 231 cells following treatment with salidroside for $24 \mathrm{~h}$ (Fig. 5A). The results suggested that salidroside dose-dependently inhibited the migratory ability of MDA-MB 231 cells (Fig. 5B). To assess whether salidroside could inhibit cell invasion, a Matrigel invasion assay (Fig. 5C) was conducted, which demonstrated that salidroside treatment decreased the relative cell invasion (Fig. 5D). Invasion was largely dependent on the release of MMPs, and salidroside downregulated MMP2, MMP3 and
MMP9 expression at both the translational (Fig. 5E) and transcriptional levels (Fig. 5F). Overall, these results confirmed that salidroside inhibited cell migration and invasion by inhibiting STAT3 phosphorylation and MMP2 expression.

\section{Discussion}

Previous studies have extensively investigated the effects of salidroside on cancer cells, and a vast body of research exists on breast cancer, including studies regarding cell cycle inhibition and mammalian target of rapamycin molecular pathway analysis $(14-16,21,22)$. However, despite work in these areas, only scarce data are available regarding the specific roles of salidroside with regards to breast cancer growth, progression and metastasis. The present study aimed to elucidate the mechanism underlying the ability of salidroside to inhibit tumor progression and metastasis by targeting EGFR signaling and its downstream molecule STAT3 as a specific target.

The results provided evidence to suggest that salidroside, extracted from $R$. rosea, inhibited the growth of breast cancer cells in vitro. Specifically, $40 \mu \mathrm{M}$ salidroside killed $49 \%$ of MDA-MB 231 TNBC cells. The observed cytotoxic effect was concentration-dependent. Conversely, salidroside exhibited no 
cytotoxic effect on MCF-10A and HUVECs, which indicated that salidroside may have no side effects on normal cells. These results suggested that salidroside does not induce cell death in normal cell lines, whereas it may induce cancer cell death, and therefore may be considered a good candidate as an anticancer drug. These in vitro data strongly suggested that salidroside is a candidate chemotherapeutic agent for breast cancer treatment.

A recent report demonstrated that salidroside significantly and dose-dependently suppresses MMP2 and MMP9 activity, and decreases protein expression in MCF-7 cells (23). This is consistent with the present findings, which indicated that salidroside treatment reduced MMP2 and MMP9 expression at the mRNA and protein levels in MDA-MB 231 cells. It has also been demonstrated that MMP2 and MMP9 are associated with the Jak2/STAT3 pathway $(20,24,25)$. STAT3 phosphorylation provokes homo- or heterodimerization, which is followed by nuclear translocation and binding to gene-specific response elements in target gene promoters (26). Together with these data, the present experimental results suggested that treatment with $40 \mu \mathrm{M}$ salidroside resulted in a reduction of binding site activity. Examination of the MMP2 gene promoter sequence revealed a GAS element in the transcription start site, and gel shift analyses confirmed that STAT3 possessed DNA-binding activity to the MMP2 gene promoter, which was inhibited by salidroside. Furthermore, STAT3 knockdown effectively eliminated MMP2 expression, whereas STAT3 overexpression led to the same pattern of STAT3 and MMP2 reduction following salidroside treatment. Finally, it was demonstrated that salidroside treatment markedly inhibited cell migration and invasion in MDA-MB 231 cells. However, the results of a wound-healing assay indicated that the inhibitory effect of salidroside on migration may be due to cell toxicity. In order to confirm this effect was not due to cell death, a Matrigel invasion assay was performed; therefore, the contact between cells and salidroside occurred through Matrigel, clearly indicating that cell death had no role in the invasion process. These findings confirmed that salidroside induced inhibition of tumor migration and invasion, which was not caused by cell toxicity, thus indicating the ability of salidroside to inhibit metastasis.

The present experimental results are consistent with the previously reported roles of Jak2 and STAT3. The Jak/STAT pathway has important signaling roles in cancer growth and progression, and has been implicated in numerous cancer types, including human colon cancer (12), breast cancer (23), melanoma (27), osteosarcoma (28), gastric cancer (21), and head and neck cancer (29). Jak proteins are implicated in cell proliferation, migration and apoptosis; they bind with and promote the phosphorylation of downstream molecules, including STAT3. Upon phosphorylation, STAT3 is activated and regulates a broad range of downstream target genes, mostly associated with malignant transformation and metastasis (30-32).

The preesnt study also observed that salidroside exerted an inhibitory effect on angiogenesis in HUVECs. Therefore, the protein expression levels of VEGF in HUVECs and MDA-MB 231 cells were detected. VEGF is considered a key endothelial cell-specific signaling factor required for tumor angiogenesis (33). In the present study, VEGF expression was reduced in HUVECs following treatment with salidroside. Previously, Ariyanti et al (34) and Zhang et al (35) reported an elevation in the expression of VEGF in salidroside-treated skeletal muscle cells compared with in non-treated cells, which indicated that angiogenesis activity is dependent on the upregulation of VEGF-A and platelet-derived growth factor-BB, thus suggesting that it is independent of VEGF downregulation. However, in the present study, salidroside inhibited angiogenesis by inhibiting VEGF activity, thus suggesting that salidroside inhibited VEGF-dependent angiogenesis. Since angiogenesis can foster tumor progression and metastasis, it is an important consideration in developing anticancer therapy (36). The present study revealed that salidroside treatment inhibited in vitro angiogenesis in endothelial cells, and led to reduced VEGF protein expression in breast cancer cells, thus suggesting the anti-angiogenic ability of salidroside. Further experimentation in a breast cancer xenograft model is required to confirm the roles of these signaling molecules in salidroside-induced inhibition of migration, invasion and angiogenesis.

Overall, it appears that salidroside treatment may inhibit STAT3 phosphorylation (in a manner involving EGFR and Jak2), which in turn inhibited STAT3 signaling by blocking STAT3 nuclear translocation and DNA-binding. This may lead to inhibition of the gene-specific transcriptional activation of MMP2, ultimately resulting in inhibition of viability, migration, invasion and angiogenesis of breast cancer cells.

In conclusion, the present data revealed that salidroside inhibited TNBC tumor migration and invasion by regulating EGFR/Jak2/STAT3 signaling in a manner that inhibited MMP2 transcription. Additionally, it was demonstrated that salidroside inhibited VEGF-dependent tumor angiogenesis via a mechanism that also involves STAT3.

\section{Acknowledgements}

Not applicable.

\section{Funding}

The present study received funding from Konkuk University in 2017.

\section{Availability of data and materials}

All data generated or analyzed during this study are included in this published article.

\section{Authors' contributions}

DYK conceived and designed the experiments, performed the experiments and wrote the paper. YMY contributed in designing the experiments and data analysis. YHJ, NSP, DHK, HGL and YMP analyzed experiments and data along with YMY. All authors contributed to revise the manuscript and approved the final version for publication.

\section{Ethics approval and consent to participate}

Not applicable. 


\section{Consent for publication}

Not applicable.

\section{Competing interests}

The authors declare that they have no competing interests.

\section{References}

1. Cleator S, Heller W and Coombes RC: Triple-negative breast cancer: Therapeutic options. Lancet Oncol 8: 235-244, 2007.

2. Anders CK and Carey LA: Biology, metastatic patterns, and treatment of patients with triple-negative breast cancer. Clin Breast Cancer 9 (Suppl 2): S73-S81, 2009.

3. Carey LA, Perou CM, Livasy CA, Dressler LG, Cowan D, Conway K, Karaca G, Troester MA, Tse CK, Edmiston S, et al: Race, breast cancer subtypes, and survival in the Carolina Breast Cancer Study. JAMA 295: 2492-2502, 2006.

4. Potemski P, Kusinska R, Watala C, Pluciennik E, Bednarek AK and Kordek R: Prognostic relevance of basal cytokeratin expression in operable breast cancer. Oncology 69: 478-485, 2005.

5. Dent R, Trudeau M, Pritchard KI, Hanna WM, Kahn HK, Sawka CA, Lickley LA, Rawlinson E, Sun P and Narod SA: Triple-negative breast cancer: Clinical features and patterns of recurrence. Clin Cancer Res 13: 4429-4434, 2007.

6. Masuda H, Zhang D, Bartholomeusz C, Doihara H, Hortobagyi GN and Ueno NT: Role of epidermal growth factor receptor in breast cancer. Breast Cancer Res Treat 136: 331-345, 2012

7. Furth PA: STAT signaling in different breast cancer sub-types. Mol Cell Endocrinol 382: 612-615, 2014.

8. Bowman T, Garcia R, Turkson J and Jove R: STATs in oncogenesis. Oncogene 19: 2474-2488, 2000.

9. Darnell JE Jr: STATs and gene regulation. Science 277: $1630-1635,1997$.

10. Thomas SJ, Snowden JA, Zeidler MP and Danson SJ: The role of JAK/STAT signalling in the pathogenesis, prognosis and treatment of solid tumours. Br J Cancer 113: 365-371, 2015

11. Wang J, Li JZ, Lu AX, Zhang KF and Li BJ: Anticancer effect of salidroside on A549 lung cancer cells through inhibition of oxidative stress and phospho-p38 expression. Oncol Lett 7: 1159-1164, 2014

12. Sun KX, Xia HW and Xia RL: Anticancer effect of salidroside on colon cancer through inhibiting JAK2/STAT3 signaling pathway. Int J Clin Exp Pathol 8: 615-621, 2015.

13. Zhang Y, Yao Y, Wang H, Guo Y, Zhang $H$ and Chen L: Effects of salidroside on glioma formation and growth inhibition together with improvement of tumor microenvironment. Chin J Cancer Res 25: 520-526, 2013.

14. Hu X, Zhang X, Qiu S, Yu D and Lin S: Salidroside induces cellcycle arrest and apoptosis in human breast cancer cells. Biochem Biophys Res Commun 398: 62-67, 2010.

15. Hu X, Lin S, Yu D, Qiu S, Zhang X and Mei R: A preliminary study: The anti-proliferation effect of salidroside on different human cancer cell lines. Cell Biol Toxicol 26: 499-507, 2010.

16. Liu Z, Li X, Simoneau AR, Jafari M and Zi X: Rhodiola rosea extracts and salidroside decrease the growth of bladder cancer cell lines via inhibition of the mTOR pathway and induction of autophagy. Mol Carcinog 51: 257-267, 2012.

17. Trott $\mathrm{O}$ and Olson AJ: AutoDock Vina: Improving the speed and accuracy of docking with a new scoring function, efficient optimization, and multithreading. J Comput Chem 31: 455-461, 2010.
18. Hofmann HD and Kirsch M: JAK2-STAT3 signaling: A nove function and a novel mechanism. JAK-STAT 1: 191-193, 2012.

19. Looyenga BD, Hutchings D, Cherni I, Kingsley C, Weiss GJ and Mackeigan JP: STAT3 is activated by JAK2 independent of key oncogenic driver mutations in non-small cell lung carcinoma. PLoS One 7: e30820, 2012.

20. Byun HJ, Darvin P, Kang DY, Sp N, Joung YH, Park JH, Kim SJ and Yang YM: Silibinin downregulates MMP2 expression via Jak2/STAT3 pathway and inhibits the migration and invasive potential in MDA-MB-231 cells. Oncol Rep 37: 3270-3278, 2017.

21. Kisseleva T, Bhattacharya S, Braunstein J and Schindler CW: Signaling through the JAK/STAT pathway, recent advances and future challenges. Gene 285: 1-24, 2002.

22. Zhang J, Liu A, Hou R, Zhang J, Jia X, Jiang W and Chen J: Salidroside protects cardiomyocyte against hypoxia-induced death: A HIF-1 $\alpha$-activated and VEGF-mediated pathway. Eur J Pharmacol 607: 6-14, 2009.

23. Zhao G, Shi A, Fan Z and Du Y: Salidroside inhibits the growth of human breast cancer in vitro and in vivo. Oncol Rep 33: 2553-2560, 2015

24. Björklund $\mathrm{M}$ and Koivunen E: Gelatinase-mediated migration and invasion of cancer cells. Biochim Biophys Acta 1755: 37-69, 2005.

25. Xie TX, Wei D, Liu M, Gao AC, Ali-Osman F, Sawaya R and Huang S: Stat3 activation regulates the expression of matrix metalloproteinase-2 and tumor invasion and metastasis. Oncogene 23: 3550-3560, 2004.

26. Darvin P, Baeg SJ, Joung YH, Sp N, Kang DY, Byun HJ, Park JU and Yang YM: Tannic acid inhibits the Jak2/STAT3 pathway and induces G1/S arrest and mitochondrial apoptosis in YD-38 gingival cancer cells. Int J Oncol 47: 1111-1120, 2015.

27. Nam S, Xie J, Perkins A, Ma Y, Yang F, Wu J, Wang Y, Xu RZ, Huang W, Horne DA, et al: Novel synthetic derivatives of the natural product berbamine inhibit Jak2/Stat3 signaling and induce apoptosis of human melanoma cells. Mol Oncol 6 : 484-493, 2012

28. Liu Y, Wang L, Wu Y, Lv C, Li X, Cao X, Yang M, Feng D and Luo Z: Pterostilbene exerts antitumor activity against human osteosarcoma cells by inhibiting the JAK2/STAT3 signaling pathway. Toxicology 304: 120-131, 2013.

29. Henson ES and Gibson SB: Surviving cell death through epidermal growth factor (EGF) signal transduction pathways: Implications for cancer therapy. Cell Signal 18: 2089-2097, 2006.

30. Siveen KS, Sikka S, Surana R, Dai X, Zhang J, Kumar AP, Tan BK, Sethi G and Bishayee A: Targeting the STAT3 signaling pathway in cancer: Role of synthetic and natural inhibitors. Biochim Biophys Acta 1845: 136-154, 2014.

31. Yu H, Lee H, Herrmann A, Buettner R and Jove R: Revisiting STAT3 signalling in cancer: New and unexpected biological functions. Nat Rev Cancer 14: 736-746, 2014

32. Darvin P, Joung YH, S P N, Kang DY, Byun HJ, Hwang DY, Cho KH, Park KD, Lee HK and Yang YM: SP N, Kang DY, Byun HJ, Hwang DY, Cho KH, Park KD, Lee HK and Yang YM: Sorghum polyphenol suppresses the growth as well as metastasis of colon cancer xenografts through co-targeting jak2/STAT3 and PI3K/Akt/mTOR pathways. J Funct Foods 15: 193-206, 2015.

33. McMahon G: VEGF receptor signaling in tumor angiogenesis. Oncologist 5 (Suppl 1): 3-10, 2000.

34. Ariyanti AD, Sisjayawan J, Zhang J, Zhang JQ, Wang GX, Miyagishi M, Wu SR and Kasim V: Elevating VEGF-A and PDGF-BB secretion by salidroside enhances neoangiogenesis in diabetic hind-limb ischemia. Oncotarget 8: 97187-97205, 2017.

35. Zhang J, Liu A, Hou R, Zhang J, Jia X, Jiang W and Chen J. Salidroside protects cardiomyocyte against hypoxia-induced death: a HIF-1alpha-activated and VEGF-mediated pathway. Eur J Pharmacol 607: 6-14, 2009.

36. Weis SM and Cheresh DA: Tumor angiogenesis: Molecular pathways and therapeutic targets. Nat Med 17: 1359-1370, 2011. 